Canad. Math. Bull. Vol. 19 (4), 1976

\title{
INDUCTIVE LIMITS OF BANACH SPACES
}

\author{
BY \\ STEVEN F. BELLENOT
}

\begin{abstract}
If $X$ and $Y$ are infinite-dimensional Banach spaces, then $Y$ is the inductive limit of Banach spaces each isomorphic to $X$.
\end{abstract}

Several authors ([1], [2], and [3]) have obtained the result that every Banach space is an inductive limit of Hilbert spaces. Valdivia [5] has shown, that if the Banach space $E$ has a weak-star separable dual, then every Banach space is an inductive limit of spaces isomorphic to $E$. The purpose of this note is to remove the restriction that $E$ has a weak-star separable dual. We need the following facts.

Fact 1. If $X$ is an infinite-dimensional Banach space, then there are

$$
\left\{x_{n}\right\} \subset X, \quad\left\{f_{n}\right\} \subset X^{*} \quad \text { with } \quad\left\|f_{n}\right\|=1, \quad f_{n}\left(x_{m}\right)=\delta_{n m}
$$

and $\left\{\left\|x_{n}\right\|\right\}$ bounded. ([4], p. 10.)

Fact 2. A linear functional $F$, on $Y$, is in $Y^{*}$ if and only if $\left\{F\left(y_{n}\right)\right\}$ is bounded for each $\left\{y_{n}\right\} \subset Y$ with $\sum_{n}\left\|y_{n}\right\|<\infty$. (If $\|F\|$ is not finite one can find $\left(\hat{y}_{n}\right)$ with $\left\|\hat{y}_{n}\right\| \leq 1$ and $\left|F\left(y_{n}\right)\right| \geq 4^{n}$, from which the Fact follows with $y_{n}=\hat{y}_{n} / 2^{n}$.)

THEOREM. If $X$ is an infinite-dimensional Banach space and $Y$ is a Banach space, then $Y$ is the inductive limit of spaces isomorphic to $X$.

Proof. Let $A$ be the set of continuous linear maps from $X$ to $Y$. Let $\xi$ (respectively $\eta$ ) be the norm topology (respectively, the inductive limit topology for the maps $T: X \rightarrow Y, T \in A$ ) on $Y$. By definition of $\eta, \xi \leq \eta$. To show the reverse inequality, we show $(Y, \eta)$ has the same dual as $(Y, \xi)$ and invoke the barreledness of $\xi$.

Suppose $F$ is a linear function on $Y$ which is not in $Y^{*}$. By Fact 2, there is $\left\{y_{n}\right\} \subset Y$ with $\sum_{n}\left\|y_{n}\right\|<\infty$, and $F\left(y_{n}\right) \rightarrow \infty$. Let $\left\{x_{n}\right\},\left\{f_{n}\right\}$ be as in Fact 1 , then the map $T: X \rightarrow Y$ given by $T(x)=\sum_{n} f_{n}(x) y_{n}$ is in $A$. Furthermore, $F T\left(x_{n}\right)=$ $F\left(y_{n}\right) \rightarrow \infty$, and $F$ does not belong to the dual of $(Y, \eta)$.

REMARK. It suffices to use the nuclear maps for $A$, rather than all of the continuous linear maps.

Received by the editors April 7, 1976 and, in revised form, May 13, 1976. 


\section{REFERENCES}

1. S. F. Bellenot, Prevarieties and intertwined completeness of locally convex spaces. Math. Ann. 217 (1975), 59-67.

2. H. Hogbe-Nlend, Topologies et bornologies nucleáires associes applications, Ann. Inst. Fourier, Grenoble 23(4) (1973), 89-104.

3. H. Jarchow and J. Swart, On Mackey convergence in locally convex spaces, Israel J. Math. 16 (1973), 150-158.

4. J. Lindenstrauss and L. Tzafriri, Classical Banach spaces, Berlin-Heidelberg-New York: Springer 1973.

5. M. Valdivia, A class of precompact sets in Banach spaces, J. Reine Angew. Math. 276 (1975), $130-136$.

Department of Mathematics

Florida STATE UnIVERSity

Tallahassee, Florida 32306

U.S.A. 